\title{
Investigation of DKP equation for spin-zero system in the presence of Gödel-type background space-time
}

\author{
Hassan Hassanabadi ${ }^{1, \mathrm{a}}$, Won Sang Chung ${ }^{2, \mathrm{~b}}$, Soroush Zare ${ }^{1, \mathrm{c}}$, Hadi Sobhani ${ }^{1, \mathrm{~d}}$ \\ ${ }^{1}$ Faculty of Physics, Shahrood University of Technology, P. O. Box 3619995161-316, Shahrood, Iran \\ ${ }^{2}$ Department of Physics and Research Institute of Natural Science, College of Natural Science, Gyeongsang National University, Jinju 52828, \\ Korea
}

Received: 11 June 2017 / Accepted: 18 January 2018 / Published online: 29 January 2018

(C) The Author(s) 2018. This article is an open access publication

\begin{abstract}
This paper contains a discussion of a relativistic spin-0 system in the presence of a Gödel-type background space-time. The Duffin-Kemmer-Petiau (DKP) equation in the presence of a Gödel-type background space-time is studied in detail. After a derivation of the final form of this equation in the considered framework, free spin- 0 particles have been studied.
\end{abstract}

\section{Introduction}

The properties of Gödel-type problem space-time have been studied in the Riemannian Gödel-type [1] and for RiemannCartan-Gödel-type space-time [2-4]. Santos et al. discussed homogeneous Gödel-type solutions in hybrid metric-Palatini gravity. They showed that under certain conditions on the matter sources the problem of finding space-time homogeneous solutions in $f(R)$ (hybrid metric-Palatini) theories reduces to the problem of determining solutions of this type in $f(R)$ gravity in the metric formalism. Also they determined a perfect-fluid Gödel-type solution in $f(R)$ gravity, and showed that it is isometric to the Gödel geometry, and therefore exhibits violation of causality [5]. Furtado et al. investigated the Gödel solution in modified gravity whose action presents itself as a sum of the usual Einstein-Hilbert action and the gravitational Chern-Simons term and showed that the Gödel metric solves the modified equations of motion [6]. As an application of Gödel-type problems in the relativistic studied we can indicate that gravitational coupling of Klein-Gordon and Dirac fields to matter vorticity and spacetime torsion, in the context of Einstein-Cartan theory with

\footnotetext{
a e-mail: hha1349@gmail.com

be-mail: mimip44@ naver.net

c e-mail: soroushzrg@gmail.com

de-mail: hadisobhani8637@gmail.com
}

a Gödel-type metric, have been done by Figueiredo [7]. A close relation was seen between a family of Gödel-type solutions of $3+1$ general relativity and the Landau problem, as reported by Drukker [8]. The quantum dynamics of a scalar particle in three spaces whose metrics are described by different classes of Gödel solutions, with a cosmic string passing through the spaces has been studied [9]. The solutions of the Klein-Gordon equation in the background of KerrNewman, Gödel and Friedmann-Robertson-Walker spacetimes with a cosmic string passing through them have been obtained; some of their consequences have been discussed, with emphasis on the role played by the presence of the cosmic string [10]. Relativistic bound states solutions for the Dirac equation dealing with three cases of the Gödel-type solutions with torsion, where a cosmic string passes through these three cases of the space-time, have been investigated [11]. Weyl fermions in a family of Gödel-type geometries in Einstein general relativity have been studied recently [12], and some valuable efforts are available in Refs. [13-16].

In recent years there has been a renewed interest in the Duffin-Kemmer-Petiau (DKP) theory describing spin-0 and spin-1 mesons [17] due to discovery of a new conserved fourvector current with positive zeroth component $[18,19]$, which can be thus interpreted as a probability density. DKP equation is similar to Dirac equation for spin-1/2 particles, because DKP is a first-order formalism like the Dirac formalism [2024]. In other words, the DKP equation is a generalized Dirac equation for integral spin interactions, which can be obtained by substituting gamma matrices by beta matrices and following an interesting algebra like the DKP algebra. In some cases it can be shown that using the DKP equation is better than the Klein-Gordon or Proca equation [25-30]. The first-order relativistic DKP equation has been used to study the interactions of spinless mesons with nuclei [31]. There have been made valuable efforts using the DKP equation such as deuteronnucleus scattering using the DKP equation, motivated by the 
fact that this theory suggests a spin-1 structure from combining two spin- $\frac{1}{2}$ [32]: study of the meson-nuclear interaction and the relativistic model of $\alpha$-nucleus elastic scattering $[33,34]$, investigation of the DKP equation, with equally vector and scalar potentials for the general deformed Morse potential, obtaining creation and annihilation operators that showed these operators satisfied the commutation relation of the $S U(1,1)$ group [35], study of the DKP equation in the presence of external electromagnetic fields in FriedmannRobertson-Walker metric [36] and with a time-dependent interaction [37].

For a free particle whose spin is 0, the well-known firstorder relativistic DKP equation is [37]

$\left(i \beta^{\mu} \partial_{\mu}-m\right) \Psi=0 \quad(\hbar=c=1)$,

where $m$ is mass of the particle and $\beta^{j}(j=0,1,2,3)$ are matrices which satisfy the commutation relation

$\beta^{\mu} \beta^{v} \beta^{\lambda}+\beta^{\lambda} \beta^{v} \beta^{\mu}=g^{\mu \nu} \beta^{\lambda}+g^{\lambda v} \beta^{\mu}$,

where Eq. (1.2) defines the DKP algebra and the metric tensor is $g^{\mu \nu}=\operatorname{diag}(1,-1,-1,-1)$. The $\beta$ matrices for the spin0 systems are

$\beta^{0}=\left(\begin{array}{cc}\theta & \overline{0} \\ \overline{0}_{T} & \mathbf{0}\end{array}\right), \quad \beta^{i}=\left(\begin{array}{cc}\tilde{0} & \rho^{i} \\ -\rho_{T}^{i} & \mathbf{0}\end{array}\right)$,

in which $\tilde{0}, \overline{0}$ and $\mathbf{0}$ are $2 \times 2,2 \times 3$ and $3 \times 3$ matrices, respectively, and also we have

$\theta=\left(\begin{array}{ll}0 & 1 \\ 1 & 0\end{array}\right), \quad \rho^{1}=\left(\begin{array}{ccc}-1 & 0 & 0 \\ 0 & 0 & 0\end{array}\right)$

$\rho^{2}=\left(\begin{array}{ccc}0 & -1 & 0 \\ 0 & 0 & 0\end{array}\right), \quad \rho^{3}=\left(\begin{array}{ccc}0 & 0 & -1 \\ 0 & 0 & 0\end{array}\right)$.

In this case $\Psi$ is a five component spinor.

In this paper, we want to study the DKP equation for spin- 0 systems in the presence of a Gödel-type background spacetime without considering torsion effects and to investigate in detail the effect of the existence of such a background on the considered system. This paper has been organized as follows: the Gödel-type background space-time and derivation of the $\beta$ matrices are presented in Sect. 2. Free spin-0 particles in the presence of Gödel-type background space-time is studied in Sect. 3. Finally, the conclusions appear.

\section{Derivation of DKP elements in the presence of Gödel-type background space-time}

In this section, it is supposed that DKP equation for spin-0 particles to be derived in the Gödel-type background space- time. At first three class of this kind of space-time will be introduced then Christoffel symbols, spin connections and spinorial affine connections will be calculated in order to construct the new $\beta$ matrices in a curve space.

The space-time metric representing a class of the Gödeltype solution with can be written as

$$
\begin{aligned}
\mathrm{d} s^{2}= & -\left(\mathrm{d} t+\alpha \Omega \frac{\sinh ^{2}(l r)}{l^{2}} \mathrm{~d} \phi\right)^{2} \\
& +\alpha^{2} \frac{\sinh ^{2}(2 l r)}{4 l^{2}}+\mathrm{d} r^{2}+\mathrm{d} z^{2},
\end{aligned}
$$

where the coordinates $(t, r, \phi, z)$ are defined in the ranges of $0 \leq r<\infty, 0 \leq \phi \leq 2 \pi$ and $-\infty<z<\infty$. Moreover, the parameter $\Omega$ characterizes the vorticity of the space-time, and the parameter $\alpha$ characterizes the cosmic string, since it is associated with the angle deficit $\alpha=(14 \lambda)$, with $\lambda$ being the mass per unit length of the cosmic string, and it assumes values in the range $0<\alpha<1$. It is instructive to note here that in the case of a Gödel-type background three classes of families of solutions are possible:

- Considering $l=0$ in the line element (2.1), then the Gödel-type space-time reduces to the Som-Raychaudhuri solution [38] in the presence of a cosmic string. Thereby, the line element becomes

$$
\mathrm{d} s^{2}=-\left(\mathrm{d} t+\alpha \Omega r^{2} \mathrm{~d} \phi\right)^{2}+\mathrm{d} r^{2}+\alpha^{2} r^{2} \mathrm{~d} \phi^{2}+\mathrm{d} z^{2} .
$$

- In the case $l^{2}<0$ in the line element (2.1), which corresponds to the Gödel-type space-time with a spherical symmetry in the presence of a cosmic string. In this case, we have the new coordinates $R=\frac{i}{2 l}$ and $\theta=\frac{r}{R}$ [9]; then the line element (2.1) becomes

$$
\begin{aligned}
\mathrm{d} s^{2}= & -\left(\mathrm{d} t+\frac{\alpha \Omega r^{2}}{1+\frac{r^{2}}{4 R^{2}} \mathrm{~d} \phi}\right)^{2} \\
& +\left(1+\frac{r^{2}}{4 R^{2}}\right)^{-2}\left(\mathrm{~d} r^{2}+\alpha^{2} r^{2} \mathrm{~d} \phi^{2}\right)+\mathrm{d} z^{2} .
\end{aligned}
$$

- We address the case of $l^{2}>0$ in the line element (2.1), which corresponds to the hyperbolic Gödel-type spacetime with the cosmic string [9]. In this case, the line element (2.1) becomes

$$
\begin{aligned}
\mathrm{d} s^{2}= & -\left(\mathrm{d} t+\frac{\alpha \Omega r^{2}}{1-l^{2} r^{2}} \mathrm{~d} \phi\right)^{2} \\
& +\left(1-l^{2} r^{2}\right)^{-2}\left(\mathrm{~d} r^{2}+\alpha^{2} r^{2} \mathrm{~d} \phi^{2}\right) .
\end{aligned}
$$


We are interested in working on the second case. Therefore, considering $l^{2}<0$ in (2.1), which means there is a spherical symmetry in the presence of a cosmic string, and introducing the new coordinates $R=i / 2 l$ and $\theta=r / R$, the line element gets the form

$$
\begin{aligned}
\mathrm{d} s^{2}= & -\left(\mathrm{d} t+\frac{\alpha \Omega r^{2}}{\left(1+\frac{r^{2}}{4 R^{2}}\right)} \mathrm{d} \varphi\right)^{2} \\
& +\left(1+\frac{r^{2}}{4 R^{2}}\right)^{-2}\left(\mathrm{~d} r^{2}+\alpha^{2} r^{2} \mathrm{~d} \varphi^{2}\right)+\mathrm{d} z^{2}
\end{aligned}
$$

The metric tensor can be deduced in view of Eq. (2.5) to be

$$
g_{\mu \nu}(\boldsymbol{x})=\left(\begin{array}{cccc}
-1 & 0 & -\frac{\alpha \Omega r^{2}}{\left(1+\frac{r^{2}}{4 R^{2}}\right)} & 0 \\
0 & \frac{1}{\left(1+\frac{r^{2}}{4 R^{2}}\right)^{2}} & 0 & 0 \\
-\frac{\alpha \Omega r^{2}}{\left(1+\frac{r^{2}}{4 R^{2}}\right)} & 0 & \frac{\alpha^{2} r^{2}-\alpha^{2} \Omega^{2} r^{4}}{\left(1+\frac{r^{2}}{4 R^{2}}\right)^{2}} & 0 \\
0 & 0 & 0 & 1
\end{array}\right),
$$

whose inverse is

$$
\begin{aligned}
\left(g_{\mu \nu}\right)^{-1}(\boldsymbol{x}) & =g^{\mu \nu}(\boldsymbol{x}) \\
& =\left(\begin{array}{cccc}
r^{2} \Omega^{2}-1 & 0 & \frac{-\left(1+\frac{r^{2}}{4 R^{2}}\right) \Omega}{a} & 0 \\
0 & \left(1+\frac{r^{2}}{4 R^{2}}\right)^{2} & 0 & 0 \\
\frac{-\left(1+\frac{r^{2}}{4 R^{2}}\right) \Omega}{\alpha} & 0 & \frac{\left(1+\frac{r^{2}}{4 R^{2}}\right)^{2}}{\alpha^{2} r^{2}} & 0 \\
0 & 0 & 0 & 1
\end{array}\right) .
\end{aligned}
$$

Using the definition of $e_{a}^{\mu}$ and $e_{\mu}^{a}$ in the presence of the Gödeltype background, we have

$$
\begin{aligned}
& e_{\mu}^{a}(\boldsymbol{x})=\left(\begin{array}{cccc}
1 & 0 & \frac{\alpha \Omega r^{2}}{\left(1+\frac{r^{2}}{4 R^{2}}\right)} & 0 \\
0 & \frac{1}{\left(1+\frac{r^{2}}{4 R^{2}}\right)} & 0 & 0 \\
0 & 0 & \frac{\alpha r}{\left(1+\frac{r^{2}}{4 R^{2}}\right)} & 0 \\
0 & 0 & 0 & 1
\end{array}\right), \\
& e_{a}^{\mu}(\boldsymbol{x})=\left(\begin{array}{cccc}
1 & 0 & -\Omega r & 0 \\
0 & \left(1+\frac{r^{2}}{4 R^{2}}\right) & 0 & 0 \\
0 & 0 & \frac{\left(1+\frac{r^{2}}{4 R^{2}}\right)}{\alpha r} & 0 \\
0 & 0 & 0 & 1
\end{array}\right),
\end{aligned}
$$

which must satisfy

$e_{a}^{\mu}(\boldsymbol{x}) e_{\nu}^{a}(\boldsymbol{x})=\delta_{\nu}^{\mu}$,

$e_{\mu}^{a}(\boldsymbol{x}) e_{b}^{v}(\boldsymbol{x})=\delta_{b}^{a}$,

$g_{\mu \nu}=e_{\mu}^{a}(\boldsymbol{x}) e_{\nu}^{b}(\boldsymbol{x}) \eta_{a b}$,

where $\eta_{a b}=\operatorname{diag}(-1,1,1,1)$. The next step is a derivation of the related Christoffel symbols. These symbols are calculated as

$\Gamma_{\nu \lambda}^{\mu}(\boldsymbol{x})=\frac{g^{\mu \rho}(\boldsymbol{x})}{2}\left(g_{\rho \nu, \lambda}(\boldsymbol{x})+g_{\rho \lambda, \nu}(\boldsymbol{x})-g_{\nu \lambda, \rho}(\boldsymbol{x})\right)$.

Therefore the four Christoffel symbols are obtained:

$\Gamma_{i j}^{t}(\boldsymbol{x})=\left(\begin{array}{cccc}0 & \frac{4 r R^{2} \Omega^{2}}{r^{2}+4 R^{2}} & 0 & 0 \\ \frac{4 r R^{2} \Omega^{2}}{r^{2}+4 R^{2}} & 0 & \frac{4 r^{3} R^{2} \alpha \Omega\left(1+4 R^{2} \Omega^{2}\right)}{\left(r^{2}+4 R^{2}\right)^{2}} & 0 \\ 0 & \frac{4 r^{3} R^{2} \alpha \Omega\left(1+4 R^{2} \Omega^{2}\right)}{\left(r^{2}+4 R^{2}\right)^{2}} & 0 & 0 \\ 0 & 0 & 0 & 0\end{array}\right)$,

$\Gamma_{i j}^{r}(\boldsymbol{x})=\left(\begin{array}{cccc}0 & 0 & r \alpha \Omega & 0 \\ 0 & -\frac{2 r}{r^{2}+4 R^{2}} & 0 & 0 \\ r \alpha \Omega & 0 & \frac{r \alpha^{2}\left(-4 R^{2}+r^{2}\left(1+8 R^{2} \Omega^{2}\right)\right)}{r^{2}+4 R^{2}} & 0 \\ 0 & 0 & 0 & 0\end{array}\right)$,

$\Gamma_{i j}^{\varphi}(\boldsymbol{x})=\left(\begin{array}{cccc}0 & -\frac{\Omega}{r \alpha} & 0 & 0 \\ -\frac{\Omega}{r \alpha} & 0 & \frac{4 R^{2}-r^{2}\left(1+4 R^{2} \Omega^{2}\right)}{r^{3}+4 r R^{2}} & 0 \\ 0 & \frac{4 R^{2}-r^{2}\left(1+4 R^{2} \Omega^{2}\right)}{r^{3}+4 r R^{2}} & 0 & 0 \\ 0 & 0 & 0 & 0\end{array}\right)$,

$\Gamma_{i j}^{z}(\boldsymbol{x})=\left(\begin{array}{cccc}0 & 0 & 0 & 0 \\ 0 & 0 & 0 & 0 \\ 0 & 0 & 0 & 0 \\ 0 & 0 & 0 & 0\end{array}\right)$,

The spin connections can be determined using Christoffel symbols with the definition

$\omega_{\mu \bar{a} \bar{b}}(\boldsymbol{x})=\eta_{\bar{a}} \bar{c} e_{v}^{\bar{c}}(\boldsymbol{x}) e_{\bar{b}}^{\sigma}(\boldsymbol{x}) \Gamma_{\sigma \mu}^{v}-\eta_{\bar{a}} \bar{c} e_{v}^{\bar{c}}(\boldsymbol{x}) \partial_{\mu} e_{\bar{b}}^{\nu}(\boldsymbol{x})$.

Thus, the spin connections are

$\omega_{t}(\boldsymbol{x})=\left(\begin{array}{cccc}0 & 0 & 0 & 0 \\ 0 & 0 & -\Omega & 0 \\ 0 & \Omega & 0 & 0 \\ 0 & 0 & 0 & 0\end{array}\right)$,

$\omega_{r}(\boldsymbol{x})=\left(\begin{array}{cccc}0 & 0 & -\frac{4 R^{2} \Omega}{r^{2}+4 R^{2}} & 0 \\ 0 & 0 & 0 & 0 \\ -\frac{4 R^{2} \Omega}{r^{2}+4 R^{2}} & 0 & 0 & 0 \\ 0 & 0 & 0 & 0\end{array}\right)$,

$\omega_{\varphi}(\boldsymbol{x})=\left(\begin{array}{cccc}0 & \frac{4 r R^{2} \alpha \Omega}{r^{2}+\alpha R^{2}} & 0 & 0 \\ \frac{4 r R^{2} \alpha \Omega}{r^{2}+4 R^{2}} & 0 & -\frac{-4 \alpha R^{2}+\alpha r^{2}\left(1+4 R^{2} \Omega^{2}\right)}{r^{2}+4 R^{2}} & 0 \\ 0 & -\frac{4 \alpha R^{2}-\alpha r^{2}\left(1+4 R^{2} \Omega^{2}\right)}{r^{2}+4 R^{2}} & 0 & 0 \\ 0 & 0 & 0 & 0\end{array}\right)$ 
$\omega_{z}(\boldsymbol{x})=\left(\begin{array}{llll}0 & 0 & 0 & 0 \\ 0 & 0 & 0 & 0 \\ 0 & 0 & 0 & 0 \\ 0 & 0 & 0 & 0\end{array}\right)$

Also the only non-vanishing component of the spinorial affine connection $\Gamma_{\mu}(\boldsymbol{x})$, according to the definition $\Gamma_{\mu}(\boldsymbol{x})=$ $\frac{1}{2} \omega_{\mu a b}(\boldsymbol{x})\left[\beta^{a}, \beta^{b}\right]$, can be identified as

$\Gamma_{t}(\boldsymbol{x})=\left(\begin{array}{ccccc}0 & 0 & 0 & 0 & 0 \\ 0 & 0 & 0 & 0 & 0 \\ 0 & 0 & 0 & \Omega & 0 \\ 0 & 0 & -\Omega & 0 & 0 \\ 0 & 0 & 0 & 0 & 0\end{array}\right)$,

$\Gamma_{r}(\boldsymbol{x})=\left(\begin{array}{lllll}0 & 0 & 0 & 0 & 0 \\ 0 & 0 & 0 & 0 & 0 \\ 0 & 0 & 0 & 0 & 0 \\ 0 & 0 & 0 & 0 & 0 \\ 0 & 0 & 0 & 0 & 0\end{array}\right)$,

$\Gamma_{\varphi}(\boldsymbol{x})=\left(\begin{array}{ccccc}0 & 0 & 0 & 0 & 0 \\ 0 & 0 & 0 & 0 & 0 \\ 0 & 0 & 0 & \frac{\alpha\left(-4 R^{2}+r^{2}\left(1+4 R^{2} \Omega^{2}\right)\right)}{r^{2}+4 R^{2}} & 0 \\ 0 & 0 & -\frac{\alpha\left(-4 R^{2}+r^{2}\left(1+4 R^{2} \Omega^{2}\right)\right)}{r^{2}+4 R^{2}} & 0 & 0 \\ 0 & 0 & 0 & 0 & 0\end{array}\right)$,

$\Gamma_{z}(\boldsymbol{x})=\left(\begin{array}{lllll}0 & 0 & 0 & 0 & 0 \\ 0 & 0 & 0 & 0 & 0 \\ 0 & 0 & 0 & 0 & 0 \\ 0 & 0 & 0 & 0 & 0 \\ 0 & 0 & 0 & 0 & 0\end{array}\right)$

Recalling Eq. (1.3), and their general form in a curved space according to $\beta^{\mu}(x)=e_{a}^{\mu} \beta^{a}$, we have

$\beta^{t}(\boldsymbol{x})=\left(\begin{array}{ccccc}0 & 1 & 0 & r \Omega & 0 \\ 1 & 0 & 0 & 0 & 0 \\ 0 & 0 & 0 & 0 & 0 \\ -r \Omega & 0 & 0 & 0 & 0 \\ 0 & 0 & 0 & 0 & 0\end{array}\right)$,

$\beta^{r}(\boldsymbol{x})=\left(\begin{array}{ccccc}0 & 0 & -1-\frac{r^{2}}{4 R^{2}} & 0 & 0 \\ 0 & 0 & 0 & 0 & 0 \\ 1+\frac{r^{2}}{4 R^{2}} & 0 & 0 & 0 & 0 \\ 0 & 0 & 0 & 0 & 0 \\ 0 & 0 & 0 & 0 & 0\end{array}\right)$,

$\beta^{\varphi}(\boldsymbol{x})=\left(\begin{array}{ccccc}0 & 0 & 0 & -\frac{1+\frac{r^{2}}{4 R^{2}}}{\alpha r} & 0 \\ 0 & 0 & 0 & 0 & 0 \\ 0 & 0 & 0 & 0 & 0 \\ \frac{1+\frac{r^{2}}{4 R^{2}}}{\alpha r} & 0 & 0 & 0 & 0 \\ 0 & 0 & 0 & 0 & 0\end{array}\right)$

$\beta^{z}(\boldsymbol{x})=\left(\begin{array}{ccccc}0 & 0 & 0 & 0 & -1 \\ 0 & 0 & 0 & 0 & 0 \\ 0 & 0 & 0 & 0 & 0 \\ 0 & 0 & 0 & 0 & 0 \\ 1 & 0 & 0 & 0 & 0\end{array}\right)$
Now, having these elements enables us to write the DKP equation in the presence of a Gödel-type background spacetime.

\section{Free spin-0 particles in the presence of Gödel-type background space-time}

In the previous section, the DKP matrices were constructed with the aim of Christoffel symbols, spin connections and spinorial affine connections. Having the $\beta$ matrices in a curve space helps us to study of DKP equation in a curve space. The DKP equation in a curved space is given by

$\left[i \beta^{\mu}(\boldsymbol{x})\left(\partial_{\mu}+\Gamma_{\mu}(\boldsymbol{x})\right)-(M+S(r))\right] \Psi=0$,

where we have derived $\beta^{\mu}(\boldsymbol{x})$ and $\Gamma_{\mu}(\boldsymbol{x})$ in the previous section. Using the derived DKP elements in the previous section, we have

$\beta^{\mu}(\boldsymbol{x}) \Gamma_{\mu}(\boldsymbol{x})=\left(\begin{array}{ccccc}0 & 0 & -\frac{1}{r}+\frac{r}{4 R^{2}} & 0 & 0 \\ 0 & 0 & 0 & 0 & 0 \\ 0 & 0 & 0 & 0 & 0 \\ 0 & 0 & 0 & 0 & 0 \\ 0 & 0 & 0 & 0 & 0\end{array}\right)$

in which summation convection has been used. Supposing the wave function to be

$\Psi(t, r, \varphi, z)=e^{-i E t+i m \varphi+i k z}\left(\begin{array}{l}C_{1}(r) \\ C_{2}(r) \\ C_{3}(r) \\ C_{4}(r) \\ C_{5}(r)\end{array}\right)$

and $S(r)=0$, we arrive at the following coupled differential equation:

$$
\begin{aligned}
& -4 \alpha M r R^{2} C_{1}(r)+4 \alpha E r R^{2} C_{2}(r)-i \alpha r^{3} C_{3}^{\prime}(r) \\
& \quad-4 i \alpha r R^{2} C_{3}^{\prime}(r)+i \alpha C_{3}(r)\left(r^{2}-4 R^{2}\right) \\
& \quad+4 \alpha E r^{2} R^{2} \Omega C_{4}(r)+m r^{2} C_{4}(r) \\
& \quad+4 m R^{2} C_{4}(r)+4 \alpha k r R^{2} C_{5}(r)=0, \\
& E C_{1}(r)-M C_{2}(r)=0, \\
& -4 M R^{2} C_{3}(r)+i\left(r^{2}+4 R^{2}\right) C_{1}^{\prime}(r)=0, \\
& C_{1}(r)\left(-4 \alpha E r^{2} R^{2} \Omega-m\left(r^{2}+4 R^{2}\right)\right) \\
& -4 \alpha M r R^{2} C_{4}(r)=0,-k C_{1}(r)-M C_{5}(r)=0,
\end{aligned}
$$

in which a prime means a derivative with respect to $r$. These equations can be decoupled after a little algebra, which results 
in

$$
\begin{aligned}
& C_{2}(r)=\frac{E C_{1}(r)}{M}, \\
& C_{3}(r)=\frac{i\left(r^{2}+4 R^{2}\right) C_{1}^{\prime}(r)}{4 M R^{2}}, \\
& C_{4}(r)=-\frac{C_{1}(r)\left(4 \alpha E r^{2} R^{2} \Omega+m r^{2}+4 m R^{2}\right)}{4 \alpha M r R^{2}}, \\
& C_{5}(r)=-\frac{k C_{1}(r)}{M}
\end{aligned}
$$

and a differential equation for the first component of the wave function as

$$
\begin{aligned}
& \left(1+\frac{r^{2}}{4 R^{2}}\right)^{2}\left[\frac{\mathrm{d}^{2} C_{1}(r)}{\mathrm{d} r^{2}}+\frac{1}{r} \frac{\mathrm{d} C_{1}(r)}{\mathrm{d} r}\right] \\
& +\left[-\left(k^{2}+M^{2}-E^{2}+(E r \Omega)^{2}\right)-\frac{m^{2} r^{2}}{16 R^{4} \alpha^{2}}-\frac{m^{2}}{2 \alpha^{2} R^{2}}\right. \\
& \left.-\frac{m^{2}}{r^{2} \alpha^{2}}-\frac{E m \Omega r^{2}}{2 \alpha R^{2}}-\frac{2 m E \Omega}{\alpha}\right] C_{1}(r)=0,
\end{aligned}
$$

which is in agreement with the differential equation obtained in Ref. [9]. Thus we can obtain a new differential equation with the help of a new variable $\xi=\frac{r^{2}}{r^{2}+4 R^{2}}$ :

$$
\begin{gathered}
\xi(1-\xi) \frac{\mathrm{d}^{2} F(\xi)}{\mathrm{d} \xi^{2}}\left[\frac{|m|}{\alpha}+1\right. \\
\left.-\left(\frac{2|m|}{\alpha}+4 \Omega R^{2} E+2\right) \xi\right] \frac{\mathrm{d} F(\xi)}{\mathrm{d} \xi} \\
\times\left[\frac{m^{2}}{\alpha^{2}}+2 \Omega R^{2}\left(\frac{|m|}{\alpha}+\frac{m}{\alpha}+1\right) E\right. \\
\left.+\frac{|m|}{\alpha}-R^{2}\left(k^{2}+M^{2}\right)\right] F(\xi)=0,
\end{gathered}
$$

where $C_{1}(\xi)=(1-\xi)^{\gamma} \xi^{\beta} F(\xi), \gamma=\frac{\lambda}{2}, \lambda=\left|\frac{m}{\alpha}+4 \Omega R^{2} E\right|$, $\beta=\left|\frac{m}{2 \alpha}\right|$. Equation (3.14) is the general form of the hypergeometric equation. Therefore, a solution of Eq. (3.14) can be written as

$$
\begin{aligned}
F(\xi)= & { }_{2} F_{1}(A, B, C, \xi), \\
A= & \frac{1}{2}\left(1+\frac{2|m|}{\alpha}+4 \Omega R^{2} E\right) \\
& +\frac{1}{2} \sqrt{1+4 \Omega R^{2} E+2 \Omega R^{2}\left(\frac{|m|}{\alpha}-\frac{m}{\alpha}\right) E+4 R^{2}\left(E^{2}-k^{2}-M^{2}\right),} \\
B= & \frac{1}{2}\left(1+\frac{2|m|}{\alpha}+4 \Omega R^{2} E\right)
\end{aligned}
$$

$$
\begin{aligned}
& -\frac{1}{2} \sqrt{1+4 \Omega R^{2} E+2 \Omega R^{2}\left(\frac{|m|}{\alpha}-\frac{m}{\alpha}\right) E+4 R^{2}\left(E^{2}-k^{2}-M^{2}\right)}, \\
& C=\frac{|m|}{\alpha}+1 .
\end{aligned}
$$

The energy eigenvalue relation can be obtained:

$$
\begin{aligned}
& E_{n, m}=-\frac{ \pm \alpha \sqrt{a+b}+c_{0}}{4 \alpha R^{2}\left(4 R^{2} \Omega^{2}-1\right)} \\
& a=8 R^{2}\left(2-8 R^{2} \Omega^{2}\right)\left(\begin{array}{l}
|m|(\alpha+|m|+2 \alpha n)+ \\
\alpha^{2}\left(R^{2}\left(k^{2}+M^{2}\right)+n^{2}+n\right)
\end{array}\right)
\end{aligned}
$$

$b=R^{4} \Omega^{2}(2 \alpha+7|m|+m+8 \alpha n)^{2} ;$

$c_{0}=R^{2} \Omega(2 \alpha+7|m|+8 \alpha n)+m R^{2} \Omega$;

For each of the energy levels $E$, the set of states having the same $n$ is once again degenerate, with degenerate states being distinguished by their angular momentum $m$. However, the degeneracy of each level is now finite, there is an upper bound and a lower bound defined by the interval $-n \leq \frac{m}{\alpha} \leq 4 R^{2} \Omega E$. Note that the presence of topological defects reduces the degeneracy of the energy levels, since the parameter has values in the range $0<\alpha<1$. It should be noted that we have not considered the effects of torsion in our calculations [35]. To present physical interpretation of the results we should plot some figures. In Fig. 1, the probability density of $C_{1}(r)$ has been plotted. In our calculation, we considered free particle. In the Minkowski space-time it can be possible to find the free particles anywhere, but as can be shown by Fig. 1, the free particles only can exist somewhere in space, but this cannot be true for the Gödel type space-time. Treatments of the probability density in the curved space considered show that free particles are not really free and without interactions with the space-time, and they are interacting. To proceed with the interpretation of the results, we have plotted the energy of particles and anti-particles as a function of $n$ in Fig. 2. In this figure we have considered a specific value for the $\alpha$ and plotted the energy as a function of $n$. The effects of the parameter $\Omega$ can easily be seen. By increasing the value of the parameter $\Omega$, the absolute value of the energy will increase. In Fig. 3, we have shown the effect of the $\alpha$ parameter on a specific level for different values of $\Omega$. It is shown that when the $\alpha$ parameter increases, the value of the energy approaches a specific value. The important results that can be understood from these figures is that in the Minkowski spacetime for the particle and anti-particle we deal with the same energy with different sign; but in the considered space-time, we deal with different values of the energy for the particle and anti-particle. This kind of distinction caused in the new curve space. It is easy to show that by removing the curvature of space-time and leaving the Gödle type and using Minkowski space-time, we can find the same energy with different signs. 
Fig. 1 The wave function probability density in the curve space. The constant are $n=5, k=1, \alpha=0.5, \Omega=$ $0.05, R=1, M=10, m=1$

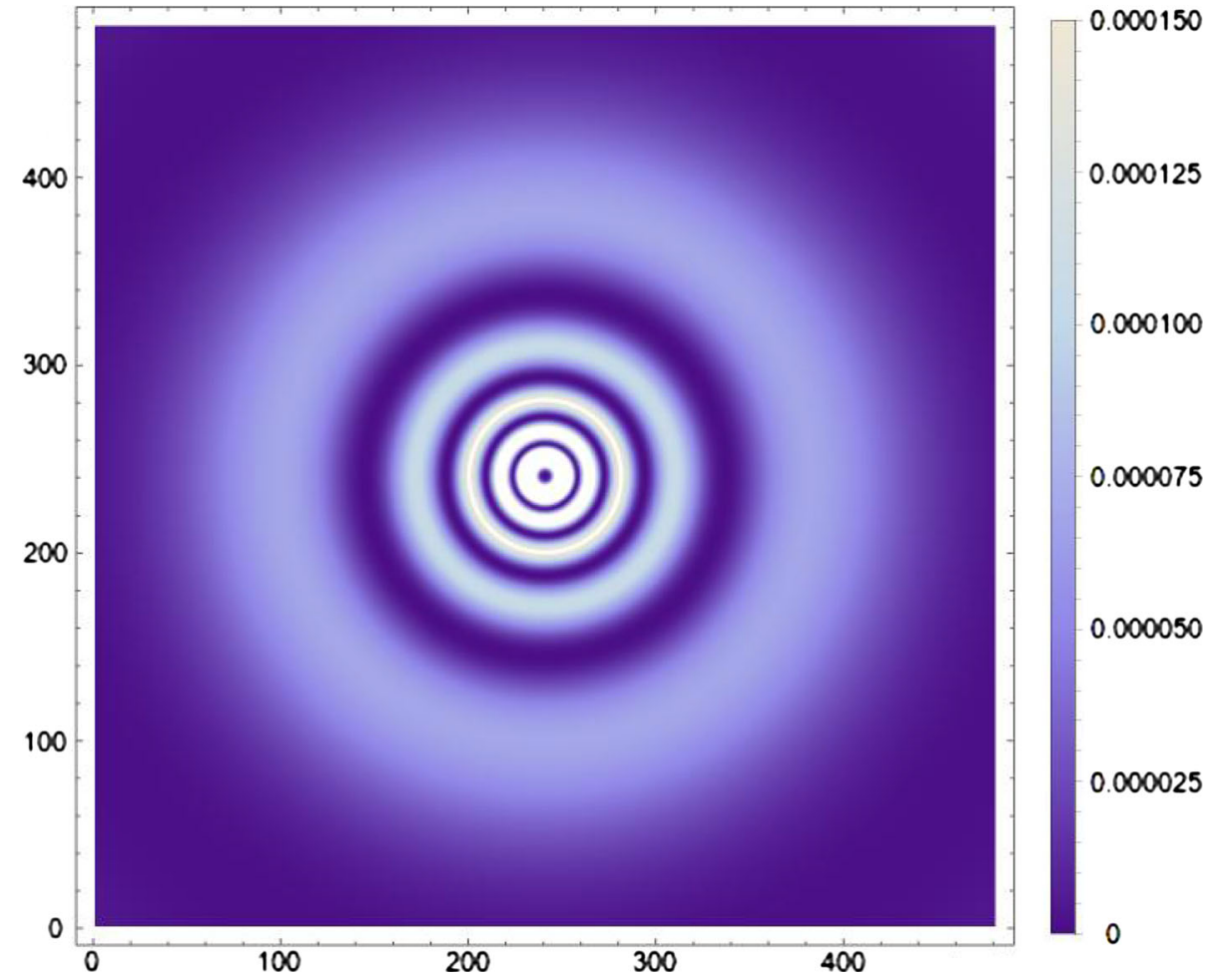

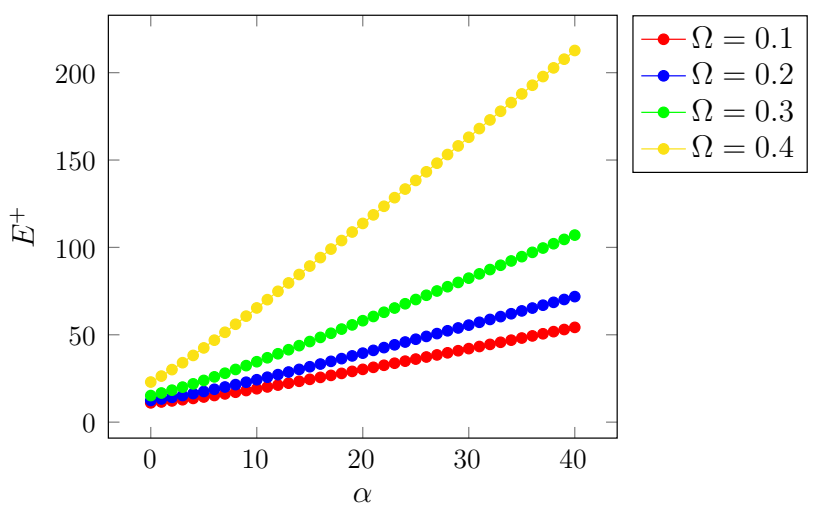

(a) Plots of $E^{+}$as function of $n$.

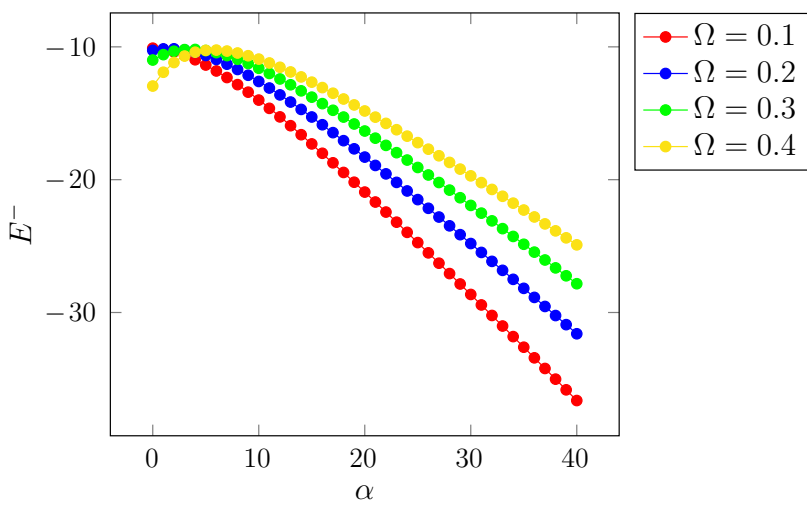

(b) Plots of $E^{+}$as function of $n$.

Fig. 2 Energy as a function of $\alpha$. The constants have been set as $\alpha=$ $0.5, k=1, R=1, M=10, m=1$

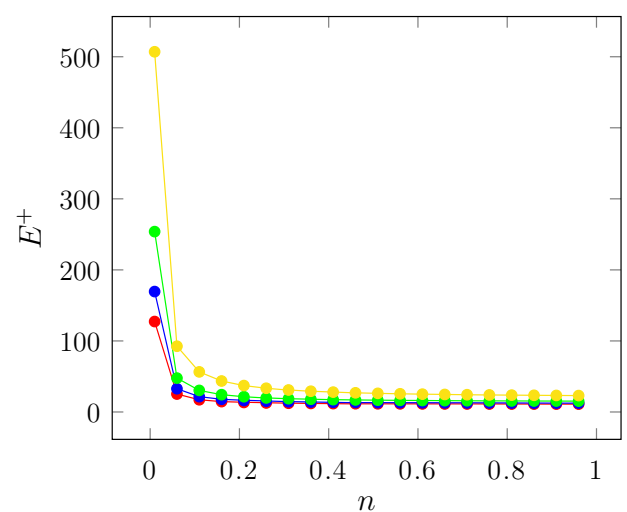

$\begin{aligned} \rightarrow \Omega & =0.1 \\ \rightarrow \Omega & =0.2 \\ -\Omega & =0.3 \\ -\Omega & =0.4\end{aligned}$

(a) Plots of $E^{+}$as function of $\alpha$.

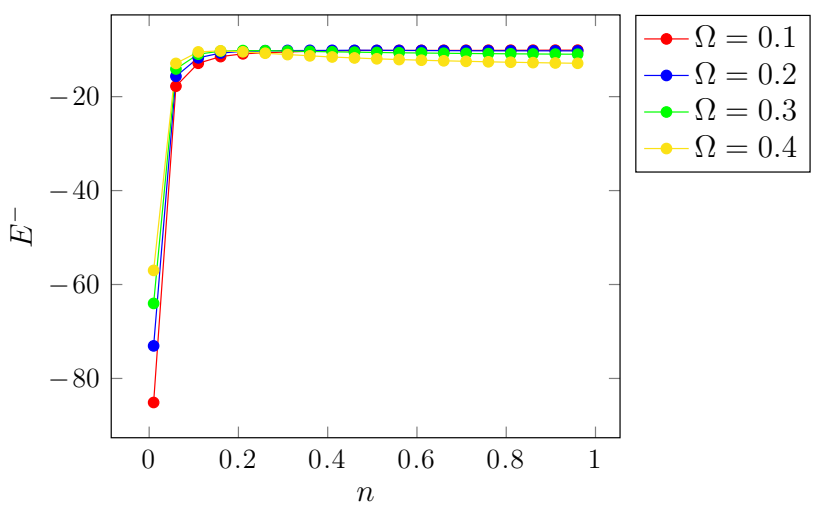

(b) Plots of $E^{+}$as function of $\alpha$.

Fig. 3 Energy as a function of $\alpha$. The constants are $\alpha=0.5, k=$ $1, R=1, M=10, m=1$ 


\section{Conclusions}

In this paper, the DKP equation for spin-0 system in the presence of Gödel-type background space-time with for free particle was studied. In Case of Gödel-type background is possible three class of family of solution. These solutions could be selected considering one of form of $l=0$ in which the Gödel-type space-time reduces to the Som-Raychaudhuri solution, $l^{2}<0$ which correspond to Gödel-type spacetime with a spherical symmetry in the presence of a cosmic string and $l^{2}>0$ that corresponds to the hyperbolic Gödel-type space-time with the cosmic string. We selected the second case for our study. The $\beta$ matrices in the Gödeltype space-time should be constructed. Consequently, we calculated Christoffel symbols, spin connections and spinorial affine connections. Having these elements and definition of covariant derivative, we could derive DKP equation in the Gödel-type space-time. Then for a free particle in that spacetime this equation was solved. The wave function and energy spectrum were derived.

Acknowledgements It is a great pleasure for the authors to thank the referee for the helpful comments.

Open Access This article is distributed under the terms of the Creative Commons Attribution 4.0 International License (http://creativecomm ons.org/licenses/by/4.0/), which permits unrestricted use, distribution, and reproduction in any medium, provided you give appropriate credit to the original author(s) and the source, provide a link to the Creative Commons license, and indicate if changes were made. Funded by SCOAP ${ }^{3}$.

\section{References}

1. M. Rebouças, J. Tiomno, Phys. Rev. D 28, 1251 (1983)

2. J.B. Fonseca-Neto, M.J. Rebouças, A.F.F. Teixeira, J. Math. Phys. 33, 2574 (1992)

3. J.B. Fonseca-Neto, M.J. Rebouças, Gen. Relativ. Gravity 30, 1301 (1998)

4. J.E. Aman, J.B. Fonseca-Neto, M.A.H. MacCallum, M.J. Rebouças, Class. Quantum Gravity 15, 1089 (1998)

5. J. Santos, M.J. Rebouças, T.B.R.F. Oliveira, A.F.F. Teixeira, arXiv: 1611.03985

6. C. Furtado et al., Phys. Rev. D 79, 124039 (2009)
7. B.D.B. Figueiredo, I.D. Soares, J. Tiomno, Class. Quantum Gravity 9, 1593 (1992)

8. N. Drukker, B. Fiol, J. Simón, JCAP 0410, 012 (2004)

9. J. Carvalho, A.M.M. de Carvalho, C. Furtado, Eur. Phys. J. C 74, 2935 (2014)

10. S.G. Fernandes, G.A. de Marques, V.B. Bezerra, Class. Quantum Gravity 23, 7063 (2006)

11. G.Q. Garcia, J.R. de Oliveira, K. Bakke, C. Furtado, Eur. Phys J. Plus 132, 123 (2017)

12. G.Q. Garcia, J.R.S. de Oliveira, C. Furtado, Int. J. Mod. Phys. D, https://doi.org/10.1142/S021827181850027X

13. C. Furtado et al., Int. J. Mod. Phys. Conf. Ser. 18, 145 (2012)

14. C. Furtado, J.R. Nascimento, AYu. Petrov, A.F. Santos, Phys. Rev. D 84, 047702 (2011)

15. J.B. Fonseca-Neto, AYu. Petrov, M.J. Rebouças, Phys. Lett. B 725, $412(2013)$

16. J.A. Agudelo et al., Phys. Lett. B 762, 96 (2016)

17. E.M. Corson, Introduction to Tensors, Spinors and Relativistic Wave Equations (relation structure) (Blackie and Son, London, 1953)

18. P. Ghose, D. Home, M.N. Sinha Roy, Phys. Lett. A 183, 267 (1993)

19. P. Ghose, D. Home, Phys. Lett. A 191, 362 (1994)

20. G. Petiau, Acad. R. Belg. A. Sci. Mém. Collect. 16(2), 1 (1936)

21. G. Petiau, Ph.D. Thesis, University of Paris (1936)

22. N. Kemmer, Proc. R. Soc. A 166, 127 (1938)

23. R.J. Duffin, Phys. Rev. 54, 1114 (1938)

24. N. Kemmer, Proc. R. Soc. A 173, 91 (1939)

25. B.C. Clark, S. Hama, G.R. Klbermann, R.L. Mercer, L. Ray, Phys. Rev. Lett. 55, 592 (1985)

26. G. Kalbermann, Phys. Rev. C 34, 2240 (1986)

27. R.E. Kozack, B.C. Clark, S. Hama, V.K. Mishra, G. Klbermann, R.L. Mercer, L. Ray, Phys. Rev. C 37, 2898 (1988)

28. R.E. Kozack, Phys. Rev. C 40, 2181 (1989)

29. V.K. Mishra, S. Hama, B.C. Clark, R.E. Kozack, R.L. Mercer, L. Ray, Phys. Rev. C 43, 801 (1991)

30. B.C. Clark, R.J. Furnstahl, L.Kurth Kerr, John Rusnak, S. Hama, Phys. Lett. B 427, 231-234 (1998)

31. Y. Nedjadi, R.C. Barrett, J. Phys. G 19, 87 (1993)

32. R.E. Kozak, B.C. Clark, S. Hama, V.K. Mishra, R.L. Mercer, L. Ray, Phys. Rev. C 40, 2181 (1989)

33. R.C. Barrett, Y. Nedjadi, Nucl. Phys. A 585, 311c (1995)

34. S. Ait-Tahar, J.S. 1-Khalili, Y. Nedjadi, Nucl. Phys. A 589, 307 (1995)

35. S. Sargolzaeipor, H. Hassanabadi, A. Boumali, Int. J. Geom. Methods Mod. Phys. (2017). https://doi.org/10.1142/ S0219887817501122

36. M. Darroodi, H. Hasaanabadi, Quantum Stud. Math. Found. (2017). https://doi.org/10.1007/s40509-017-0103-4

37. H. Sobhani, H. Hassanabadi, Phys. Part. Nuclei Lett. 14, 83 (2017)

38. M.M. Som, A.K. Raychaudhuri, Proc. R. Soc. A 3(04), 81 (1968) 